\title{
Proximity relations for real rank one valuations dominating a local regular ring
}

\begin{abstract}
We study 0-dimensional real rank one valuations centered in a regular local ring of dimension $n \geq 2$ such that the associated valuation ring can be obtained from the regular ring by a sequence of quadratic transforms. We define two classical invariants associated to the valuation (the refined proximity matrix and the multiplicity sequence) and we show that are equivalent data of the valuation.
\end{abstract}

\section{Introduction}

Let $R$ be a local noetherian regular ring of Krull dimension $n \geq 2$. Let $v$ be a real rank one valuation of the quotient field $K(R)$ of $R$ with $V$ as associated valuation ring. Let us assume that $V$ dominates $R$ and that $v$ is 0-dimensional (i.e. the field extension $R / M(R) \subset V / M(V)$ is algebraic, where $M(R)$ and $M(V)$ denotes the maximal ideals of $R$ and $V$ respectively).

In this situation, there exists one and only one sequence

$$
\text { (*) } R=R_{0} \subset R_{1} \subset \cdots \subset R_{i} \subset \cdots \subset V
$$

such that $R_{i+1}$ is the only quadratic transform of $R_{i}$ which is dominated by $V, i \geq 0$. (Note that $R_{i}$ has dimension $n$ for all $i \geq 0$ ).

If $n=2$ it is well-known (see [1]) that $\bigcup_{i \geq 0} R_{i}=V$. If $n \geq 3$, then $\bigcup_{i \geq 0} R_{i}=V$ if and only if the sequence $(*)$ switches strongly infinitely often. When $v$ is a non-discrete valuation this is proved in [13]. In fact, if the sequence $(*)$ switches strongly infinitely often the proof given in [13]

2000 Mathematics Subject Classification: Primary 13F30; Secondary 13H05.

Keywords: Valuation, real rank, regular ring, quadratic transform. 
shows that $\bigcup_{i>0} R_{i}=V$ regardless if $v$ is discrete or non-discrete. If now $V=\bigcup_{i>0} R_{i}$ is a discrete valuation ring, then we obtain that the sequence $(*)$ switches strongly infinitely often from domination and dimension of $R_{i}$. (See Theorem 6 below).

On the other hand, given the sequence $(*)$ one can define as usual when $R_{j}$ is proximate to $R_{i}$ for $j>i$. (See for example [8], [10], [11]...). This information is collected into the refined proximity matrix.

Also, other invariant associated to $(*)$ is the usual multiplicity sequence (see [11]). The aim of the paper is to prove that the refined proximity matrix and the multiplicity sequence are equivalent data associated to $(*)$, when the sequence switches strongly infinitely often. This is a classical wellknown result for $n=2$. In this case, there are many invariants associated to $(*)$ which are equivalent. (See [8], [9], [11]). All of them are invariants that characterize the equisingularity class of analytically irreducible plane curves, when the sequence $(*)$ is the resolution sequence of the corresponding singularity.

The paper is organized in six sections (including this introduction) as follows.

- Section 2 is devoted to remember some concepts, notations and properties about quadratic transforms.

- The main properties of rank one valuations centered on a local regular ring are given in section 3 .

- In sections 4 and 5 we define and study the refined proximity matrix and the multiplicity sequence for a valuation centered on a local regular ring. Also we prove our main result (Theorem 18) that asserts that these invariants are equivalent data of valuation, when the corresponding sequence $(*)$ switches strongly infinitely often.

- The last section is devoted to obtain technical results that are used in the proof of main result.

\section{Notations and preliminaries}

The main part of the concepts and notations of this paper are equal or similar to some of [2], [3], [6] and [13].

For a noetherian local ring $R$, we denote by $M(R)$ the maximal ideal of $R$, by $\operatorname{dim}(R)$ the Krull dimension of $R$ and by

$$
\mathfrak{B}(R)=\left\{R_{\mathfrak{p}} ; \mathfrak{p} \text { is a prime ideal of } R\right\}
$$


Also, for each non-zero principal ideal $J$ of $R$ we denote by $\operatorname{Ord}_{R}(J)$ the usual multiplicity, that is the non-negative integer $d$ such that $J \subset(M(R))^{d}$ and $J \not \subset(M(R))^{d+1}$.

A quadratic transform of $R$ is a ring $R_{1}=\left(R\left[z^{-1} M(R)\right]\right)_{\mathfrak{q}}$, where $z$ is a non-zero element of $M(R), z \notin M(R)^{2}$ and $\mathfrak{q}$ is a prime ideal of $R\left[z^{-1} M(R)\right]$ such that $M(R) R\left[z^{-1} M(R)\right] \subset \mathfrak{q}$.

By a hypersurface we mean a pair $(R, J)$, where $R$ is a regular noetherian local ring and $J$ is a non zero principal ideal of $R$. (Note that $R / J$ might not be a reduced ring).

Let $(R, J)$ be a hypersurface and $R_{1}$ a quadratic transform of $R$. The strict transform of $(R, J)$ in $R_{1}$ is the hypersurface $\left(R_{1}, J_{1}\right)$ where $J_{1}$ is the ideal such that $J_{1} z^{m} R_{1}=J R_{1}$ with $M(R) R_{1}=z R_{1}$ and $m=\operatorname{Ord}_{R}(J)$. The hypersurface $\left(R_{1}, J R_{1}\right)$ is called the total transform of $(R, J)$ in $R_{1}$.

Let

$$
R=R_{0} \subset R_{1} \subset \cdots \subset R_{N}
$$

be a sequence such that $R_{i}$ is a quadratic transform of $R_{i-1}, 1 \leq i \leq N$. The strict transform of a hypersurface $(R, J)$ in $R_{i}$ is the hypersurface $\left(R_{i}, J_{i}\right)$ defined recursively as follows:

1. If $i=0$, then $\left(R_{0}, J_{0}\right)=(R, J)$.

2. If $i>0$, and $\left(R_{i-1}, J_{i-1}\right)$ is the strict transform of $(R, J)$ in $R_{i-1}$, then $\left(R_{i}, J_{i}\right)$ is the strict transform of $\left(R_{i-1}, J_{i-1}\right)$ in $R_{i}$.

Now we will prove two useful results.

Lemma 1 Let $R$ be a regular noetherian local ring with $\operatorname{dim}(R)=n \geq 2$ and let $R^{\prime}$ be a quadratic transform of $R$. Then there exists a basis $\left(y_{1}, \ldots, y_{n}\right)$ of $M(R)$ such that $R^{\prime}=\left(R\left[y_{2} / y_{1}, \ldots, y_{n} / y_{1}\right]\right)_{Q}$ with $Q$ a prime ideal of $R\left[y_{2} / y_{1}, \ldots, y_{n} / y_{1}\right]$ and $M(R)\left(R\left[y_{2} / y_{1}, \ldots, y_{n} / y_{1}\right]\right) \subset Q$.

Furthermore, $R \notin \mathfrak{B}\left(R^{\prime}\right)$ and $\mathfrak{B}(R) \cap \mathfrak{B}\left(R^{\prime}\right)=\left\{S^{\prime} \in \mathfrak{B}\left(R^{\prime}\right) ; y_{1} \notin M\left(S^{\prime}\right)\right\}$. More precisely, if $\mathfrak{q}^{\prime} \in \operatorname{Spec}\left(R^{\prime}\right), y_{1} \notin M\left(R_{\mathfrak{q}^{\prime}}^{\prime}\right)$, then $R_{\mathfrak{q}^{\prime}}^{\prime}=R_{\mathfrak{q}}$ where $\mathfrak{q}:=\mathfrak{q}^{\prime} \cap R$.

Proof: The first statement is an easy consequence of the definition of quadratic transformation. So we can assume $M(R)=\left(y_{1}, \ldots, y_{n}\right)$, and

$$
R^{\prime}=\left(R\left[y_{2} / y_{1}, \ldots, y_{n} / y_{1}\right]\right)_{Q} .
$$

If $R \in \mathfrak{B}\left(R^{\prime}\right)$ then $R=\left(R^{\prime}\right)_{\mathfrak{q}^{\prime}}$ and $y_{1} \in M(R) \subset \mathfrak{q}^{\prime} R^{\prime}$. As $y_{2} / y_{1} \in R^{\prime} \subset$ $\left(R^{\prime}\right)_{\mathfrak{q}^{\prime}}=R$ and $y_{2}=\left(y_{2} / y_{1}\right) y_{1}$ then $\left(y_{1}, y_{2}, \ldots, y_{n}\right)$ is not a regular system of parameters of $R$. Thus $R \notin \mathfrak{B}\left(R^{\prime}\right)$. 
To finish the prove we will see

$$
\left\{S^{\prime} \in \mathfrak{B}\left(R^{\prime}\right) ; y_{1} \notin M\left(S^{\prime}\right)\right\}=\mathfrak{B}\left(R^{\prime}\right) \cap(\mathfrak{B}(R)-\{R\}) .
$$

Let us consider $S^{\prime} \in \mathfrak{B}\left(R^{\prime}\right)$. We can write $S^{\prime}=\left(R\left[y_{2} / y_{1}, \ldots, y_{n} / y_{1}\right]\right)_{\mathfrak{q}^{\prime}}$, with $\mathfrak{q}^{\prime}=M\left(S^{\prime}\right) \cap R\left[y_{2} / y_{1}, \ldots, y_{n} / y_{1}\right]$.

If $y_{1} \notin M\left(S^{\prime}\right)$ and $\mathfrak{q}=\mathfrak{q}^{\prime} \cap R$ then $S^{\prime}=\left(R\left[y_{2} / y_{1}, \ldots, y_{n} / y_{1}\right]\right)_{\mathfrak{q}^{\prime}}=R_{\mathfrak{q}}$.

Conversely, if $S^{\prime} \in \mathfrak{B}\left(R^{\prime}\right) \cap(\mathfrak{B}(R)-\{R\})$ we have

$$
S^{\prime}=\left(R\left[y_{2} / y_{1}, \ldots, y_{n} / y_{1}\right]\right)_{\mathfrak{q}^{\prime}}=R_{\mathfrak{q}},
$$

for some prime ideal $\mathfrak{q}$ of $R$.

Therefore, $y_{2} / y_{1}, \ldots, y_{n} / y_{1} \in R_{\mathfrak{q}}$ and $y_{1} \notin M\left(S^{\prime}\right)$. In fact, if $y_{1} \in M\left(S^{\prime}\right)$ then $y_{1} \in \mathfrak{q}=M\left(S^{\prime}\right) \cap R$. Thus we can write $y_{2} / y_{1}=f / g$ with $f, g \in R$ without common factors and $g \notin \mathfrak{q}$. So $y_{2} g=y_{1} f$ and as $y_{2} \notin y_{1} R$ we have $g \in y_{1} R \subset \mathfrak{q}$, which is a contradiction. Henceforth $y_{1} \notin M\left(S^{\prime}\right)$.

Lemma 2 Let $R$ be a regular noetherian local ring with $\operatorname{dim}(R)=n \geq 2$ and let $R_{1}$ be a quadratic transform of $R$. Let us consider $f \in M(R)$ and let us write $\left(R_{1}, f_{1} R_{1}\right)$ the strict transform of $(R, f R)$ in $R_{1}$. Let us assume that $f_{1} \in M\left(R_{1}\right)$ and $M(R)=\left(z_{1}, \ldots, z_{n}\right)$, with $M(R) R_{1}=z_{1} R_{1}$. Then we have the following statements:

1. $f_{1} \notin z_{1} R_{1}$.

2. If $f \notin z_{j} R_{1}$ and $z_{j} / z_{1} \in M\left(R_{1}\right)$ for some $2 \leq j \leq n$, then $f_{1} \notin$ $\left(z_{j} / z_{1}\right) R_{1}$.

3. If $f$ is an irreducible element of $R$, then $f_{1}$ is an irreducible element in $R_{1}$.

4. If $f$ is an irreducible element of $R$ and $f \notin z_{1} R$, then $f_{1} R_{1} \cap R=f R$.

Proof: Let us consider the ring $A:=R\left[z_{1}^{-1} M(R)\right]$ and a prime ideal $Q$ of $A$ with $M(R) A \subset Q$ and $R_{1}=R_{Q}$.

We point out that an element $r / z_{1}^{\beta} \in A, r \in M(R)^{\beta}$, is a unit in $R_{1}$ if and only if $\operatorname{Ord}_{R}(r R)=\beta$. Note that, $\operatorname{Ord}_{R}(r R) \geq \beta$ and if $\operatorname{Ord}_{R}(r R)>\beta$ then $r / z_{1}^{\beta}=\left(r / z_{1}^{\beta+1}\right) z_{1} \in M\left(R_{1}\right)$.

We can write $f_{1} R_{1}=\left(f / z_{1}^{d}\right) R_{1}$, with $\operatorname{Ord}_{R}(f R)=d$.

1) If $f_{1} \in z_{1} R_{1}$, let us write

$$
\frac{f}{\left(z_{1}\right)^{d}}=\frac{\bar{h}}{\bar{r}} z_{1}
$$

with $\bar{h}, \bar{r} \in R_{1}$ and $\bar{r} \notin M\left(R_{1}\right)$. 
In fact, $\bar{h}=h / z_{1}^{\alpha}$ and $\bar{r}=r / z_{1}^{\beta}$, with $h, r \in R, h, r \notin z_{1} R, \operatorname{Ord}_{R}(h R) \geq \alpha$ and $\operatorname{Ord}_{R}(r R)=\beta$.

Thus

$$
f r=h\left(z_{1}\right)^{d+\beta-\alpha+1} .
$$

If $d+\beta-\alpha+1 \geq 0$, then $\operatorname{Ord}_{R}(f R) \geq d+1$ and if $d+\beta-\alpha+1<0$, then $h \in z_{1} R$. So in any case we get to a contradiction and necessarily $f_{1} \notin z_{1} R_{1}$.

2) Let us assume $f \notin z_{j} R_{1}$ and $z_{j} / z_{1} \in M\left(R_{1}\right)$ for some $2 \leq j \leq n$. If $f_{1} \in\left(z_{j} / z_{1}\right) R_{1}$, we can write

$$
\frac{f}{\left(z_{1}\right)^{d}}=\frac{\bar{h}}{\bar{r}} \frac{z_{j}}{z_{1}},
$$

with $\bar{h}, \bar{r} \in R_{1}$ and $\bar{r} \notin M\left(R_{1}\right)$. As above, $\bar{h}=h / z_{1}^{\alpha}$ and $\bar{r}=r / z_{1}^{\beta}$, with $h, r \in R, h, r \notin z_{1} R, \operatorname{Ord}_{R}(h R) \geq \alpha$ and $\operatorname{Ord}_{R}(r R)=\beta$.

Thus

$$
f r=h z_{j}\left(z_{1}\right)^{d+\beta-\alpha-1} .
$$

As $f \notin z_{j} R$, then $r \in z_{j} R$. So $r=r^{\prime} z_{j}$ and

$$
\frac{r}{\left(z_{1}\right)^{\beta}}=\frac{r^{\prime}}{\left(z_{1}\right)^{\beta-1}} \frac{z_{j}}{z_{1}} \in M\left(R_{1}\right)
$$

which is a contradiction. So $f_{1} \notin\left(z_{j} / z_{1}\right) R_{1}$.

3) First we note that $f \notin z_{1} R$, because in other case $f R=z_{1} R$ and $f_{1} \notin M\left(R_{1}\right)$.

Let us assume, if it is possible, that

$$
\frac{f}{\left(z_{1}\right)^{d}}=\frac{\bar{h}_{1}}{\bar{r}_{1}} \frac{\bar{h}_{2}}{\bar{r}_{2}},
$$

with $\bar{h}_{1}, \bar{r}_{1}, \bar{h}_{2}, \bar{r}_{2} \in R_{1}$ and $\bar{r}_{1}, \bar{r}_{2} \notin M\left(R_{1}\right)$.

As always, $\bar{h}_{1}=h_{1} / z_{1}^{\alpha_{1}}, \bar{h}_{2}=h_{2} / z_{1}^{\alpha_{2}}, \bar{r}_{1}=r_{1} / z_{1}^{\beta_{1}}, \bar{r}_{2}=r_{2} / z_{1}^{\beta_{2}}$, with $h_{1}, h_{2}, r_{1}, r_{2} \in R$ and $h_{1}, h_{2}, r_{1}, r_{2} \notin z_{1} R$. Also note $\operatorname{Ord}_{R}\left(h_{i} R\right) \geq \alpha_{i}$ and $\operatorname{Ord}_{R}\left(r_{i} R\right)=\beta_{i}, 1 \leq i \leq 2$.

Thus

$$
f r_{1} r_{2}=h_{1} h_{2}\left(z_{1}\right)^{d-\alpha_{1}-\alpha_{2}+\beta_{1}+\beta_{2}} .
$$

Moreover, as $f, h_{1}, h_{2}, r_{1}, r_{2} \notin z_{1} R$ we have $d-\alpha_{1}-\alpha_{2}+\beta_{1}+\beta_{2}=0$.

Now, after removing out common factors, we can write

$$
f=h_{1}^{\prime} h_{2}^{\prime}
$$


As $f$ is an irreducible element of $R$ then, either $h_{1}^{\prime} \notin M(R)$ or $h_{2}^{\prime} \notin M(R)$. Thus, either $\bar{h}_{1} /\left(\bar{r}_{1} \bar{r}_{2}\right) \notin M\left(R_{1}\right)$ or $\bar{h}_{2} /\left(\bar{r}_{1} \bar{r}_{2}\right) \notin M\left(R_{1}\right)$ and $f_{1}$ is an irreducible element of $R_{1}$.

4) Let us consider $g \in f_{1} R_{1} \cap R$. We have

$$
g=\frac{f}{\left(z_{1}\right)^{d}} \frac{\bar{h}}{\bar{r}}
$$

with $\bar{h}, \bar{r} \in R_{1}$ and $\bar{r} \notin M\left(R_{1}\right)$. As above, $\bar{h}=h / z_{1}^{\alpha}, \bar{r}=r / z_{1}^{\beta}$, with $h, r \in R$ and $h, r \notin z_{1} R$. Also note $\operatorname{Ord}_{R}(h R) \geq \alpha$ and $\operatorname{Ord}_{R}(r R)=\beta$.

So we have

$$
f h=g r\left(z_{1}\right)^{d+\alpha-\beta} .
$$

As $f R \notin z_{1} R$, then $g r \in f R$. If $r \in f R$, then $r / z_{1}^{\beta} \in f_{1} R_{1} \subset M\left(R_{1}\right)$ which is a contradiction. So $g \in f R$.

Remark 3 Let $R=R_{0} \subset R_{1} \subset R_{2} \subset \cdots \subset R_{n} \subset \cdots$ be a sequence of regular noetherian local rings of the same dimension, such that $R_{i}$ is a quadratic transform of $R_{i-1}$, for $i \geq 1$. If $f$ is an irreducible element of $R$, $\left(R_{i}, f_{i} R_{i}\right)$ is the strict transform of $(R, f R)$ in $R_{i}$ and $f_{i} \in M\left(R_{i}\right)$, then $f_{i}$ is an irreducible element in $R_{i}$ for $i \geq 0$.

\section{Valuations of real rank one}

In this section we shall consider a local noetherian regular ring $R$ with $\operatorname{dim}(R)=n \geq 2$ and let $v$ be a valuation of the quotient field $K(R)$ of $R$ with $V$ as valuating ring. Let us assume that $V$ dominates $R$ (i.e. $R \subset V$ and $M(R)=R \cap M(V)$ ) and that $v$ is 0-dimensional (i.e. the field extension $R / M(R) \subset V / M(V)$ is algebraic).

Also, we shall consider the sequence

$$
\left(R_{i}\right) \equiv R=R_{0} \subset R_{1} \subset R_{2} \subset \cdots \subset R_{n} \subset \cdots
$$

of regular noetherian local rings of the same dimension, such that $R_{i}$ is the quadratic transform of $R_{i-1}$, along $V$ for $i \geq 1$. Note that $\operatorname{dim}\left(R_{i}\right)=$ $\operatorname{dim}\left(R_{i+1}\right)$ implies that $R_{i+1} / M\left(R_{i+1}\right)$ is a finite extension of $R_{i} / M\left(R_{i}\right)$ (because

$$
\operatorname{dim}\left(R_{i+1}\right)=\operatorname{dim}\left(R_{i}\right)+\operatorname{tr} \cdot \operatorname{deg} .\left(\left(R_{i+1} / M\left(R_{i+1}\right): R_{i} / M\left(R_{i}\right)\right)\right)
$$

(see [13], p. 296)).

Now, we point out the following definition. (See [13], p. 314). 
Definition 4 The quadratic sequence $\left(R_{i}\right)$ switches strongly infinitely often if there does not exist an integer $j$ and a height one prime ideal $\mathfrak{p}$ in $R_{j}$ with the property that

$$
\bigcup_{i \geq 0} R_{i} \subset\left(R_{j}\right)_{\mathfrak{p}}
$$

Lemma 5 With the above notations, let us assume that $\left(R_{i}\right)$ switches strongly infinitely often. Let us consider $f \in R=R_{0}$. Then there exists a nonnegative integer $j_{0} \geq 0$ such that $f_{j} R_{j}=R_{j}$ for all $j \geq j_{0}$, where $\left(R_{j}, f_{j} R_{j}\right)$ is the strict transform of $(R, f R)$ in $R_{j}$ for all $j \geq 0$.

Proof: If $f_{j} R_{j} \neq R_{j}$ for all $j \geq 0$, we can assume that $f$ is an irreducible element of $R$. Thus, by Remark $3, f_{j}$ is an irreducible element of $R_{j}$ for all $j \geq 0$.

On the other hand, let us write $M\left(R_{j}\right) R_{j+1}=z_{j} R_{j+1}$ with $z_{j} \in M\left(R_{j}\right)$ for $j \geq 0$.

Also by Lemma $2, f_{j+1} \notin z_{j} R_{j+1}, f_{j+1} R_{j+1} \cap R_{j}=f_{j} R_{j}$, and by Lemma 1 ,

$$
(R)_{f R}=\left(R_{j}\right)_{f_{j} R_{j}} \in \mathfrak{B}\left(R_{j+1}\right),
$$

for all $j \geq 0$. So $R_{j+1} \subset\left(R_{j}\right)_{f_{j} R_{j}}$ and $\bigcup_{i=1}^{\infty} R_{i} \subset(R)_{f R}$ which is a contradiction.

Theorem 6 With the above notations, let us assume that the valuation $v$ has real rank one. Then the following statements are equivalent:

1. $V=\bigcup_{i \geq 0} R_{i}$.

2. $\left(R_{i}\right)$ switches strongly infinitely often.

Proof: When $v$ is a non-discrete valuation this is proved in Proposition (4.18) of [13]. In fact, if the sequence $\left(R_{i}\right)$ switches strongly infinitely often, the proof given in [13] shows that $V=\bigcup_{i>0} R_{i}$ regardless if $V$ is discrete or non-discrete, and if $V$ is non-discrete and $V=\bigcup_{i \geq 0} R_{i}$ then $\left(R_{i}\right)$ switches strongly infinitely often.

If now $V=\bigcup_{i \geq 0} R_{i}$ is a discrete valuation ring, and if there would exist a non-negative integer $j$ and a height one prime ideal $\mathfrak{p}$ of $R_{j}$ with $V \subset\left(R_{j}\right)_{\mathfrak{p}}$, then $V=\left(R_{j}\right)_{\mathfrak{p}}$. Hence $M(V) \cap R_{j}=\mathfrak{p}$, and $V$ would not dominate $R_{j}$ (since $\operatorname{dim}\left(R_{j}\right)=n \geq 2$ ).

Remark 7 In the conditions of the above theorem, note that if $V=\bigcup_{i \geq 0} R_{i}$ then

$$
\frac{V}{M(V)}=\bigcup_{i \geq 0}\left(\frac{R_{i}}{M\left(R_{i}\right)}\right) \text {. }
$$




\section{Proximity}

In this section, let

$$
\left(R_{i}\right) \equiv R=R_{0} \subset R_{1} \subset R_{2} \subset \cdots \subset R_{n} \subset \cdots
$$

be a fixed sequence of regular noetherian local rings of the same dimension, such that $R_{i}$ is a quadratic transform of $R_{i-1}$, for $i \geq 1$.

We will write

$$
V=\bigcup_{i \geq 0} R_{i}
$$

Definition 8 With the above notations, for $j>i$ we say that $R_{j}$ is proximate to $R_{i}$ if the valuation ring $V\left(R_{i}\right)$ of $\operatorname{Ord}_{R_{i}}$ contains $R_{j}$, where $\operatorname{Ord}_{R_{i}}$ is the usual valuation order of $R_{i}$.

For $i \geq 0$, we will denote the set of proximate points of $R_{i}$ by

$$
\mathcal{P}\left(R_{i}\right)=\left\{R_{j} ; R_{j} \text { is proximate to } R_{i}\right\} .
$$

Remark 9 Note that $V\left(R_{i}\right)=\left(R_{i+1}\right)_{M\left(R_{i}\right) R_{i+1}}$. Thus if $R_{j} \subset V\left(R_{i}\right)(i<j)$ also $R_{h} \subset V\left(R_{i}\right)$ for $i<h \leq j$. So as a consequence of Lemma 1 we have

$$
V\left(R_{i}\right)=\left(R_{h}\right)_{\mathfrak{p}_{h}},
$$

where $\mathfrak{p}_{h}$ is a height one prime ideal of $R_{h}, i<h \leq j$.

In this section we will use the following notations. We will denote by

$$
E_{i}^{i+1}=\left(R_{i+1}, M\left(R_{i}\right) R_{i+1}\right)=\left(R_{i+1}, D_{i}^{i+1}\right),
$$

that is, the exceptional divisor attached to $R_{i}, i \geq 0$.

Also, we will write

$$
E_{i}^{j}=\left(R_{j}, D_{i}^{j}\right)
$$

the strict transform of $E_{i}^{i+1}$ in $R_{j}$, for $i<j$.

Lemma 10 With the above notations, the following statements are equivalent for $i<j$ :

1) $R_{j} \in \mathcal{P}\left(R_{i}\right)$

2) $D_{i}^{j} \neq R_{j}$.

Proof: It is an easy consequence of Remark 9 and Lemmas 1 and 2. 
Lemma 11 With the above notations, we have the following statements:

1) $R_{i+1} \in \mathcal{P}\left(R_{i}\right), i \geq 0$.

2) If $R_{j} \in \mathcal{P}\left(R_{i}\right)$, then $R_{h} \in \mathcal{P}\left(R_{i}\right), i<h \leq j$.

3) Assume that $V$ is the valuation ring of a rank one valuation $v$ of the quotient field of $R=R_{0}$. Then $\mathcal{P}\left(R_{i}\right)$ is a finite set, $i \geq 0$.

Proof: 1) and 2) are an easy consequence of definition of proximity. 3) It is a consequence of Lemma 5, Lemma 10, and Theorem 6.

Now we will collect the proximity relations into the refined proximity matrix in the same way as in [11].

Definition 12 With the notations as above, the refined proximity matrix associated to $\left(R_{i}\right)$ is the infinite matrix $P\left(R_{i}\right)=\left(p_{i j}\right)_{i, j \geq 0}$ given by $p_{i i}=1$,

$$
p_{i j}=-\left[R_{j} / M\left(R_{j}\right): R_{i} / M\left(R_{i}\right)\right]
$$

if $R_{j} \in \mathcal{P}\left(R_{i}\right)$ and $p_{i j}=0$ for the rest.

Note that $P\left(R_{i}\right)$ is an upper triangular matrix. When $V$ is the valuation ring of a rank one valuation $v$ of the quotient field of $R=R_{0}$, we will also write

$$
P\left(R_{i}\right)=P(V)=P_{v} .
$$

Remark 13 Note that we have the following dictionary.

1) $p_{i+1} \neq 0$, for $i \geq 0$.

2) If $p_{i j} \neq 0$, then $p_{i h} \neq 0$ for $i<h \leq j$.

3) If $V$ is the valuation ring of a rank one valuation $v$ of the quotient field of $R=R_{0}$, then for fixed $i, p_{i j}=0$ for all but finitely many index $j$.

\section{Multiplicity sequence}

As in the last section, let

$$
\left(R_{i}\right) \equiv R=R_{0} \subset R_{1} \subset R_{2} \subset \cdots \subset R_{n} \subset \cdots
$$

be a fixed sequence of regular noetherian local rings of the same dimension, such that $R_{i}$ is a quadratic transform of $R_{i-1}$, for $i \geq 1$.

We will assume that

$$
V=\bigcup_{i \geq 0} R_{i}
$$

is the ring of a rank one valuation $v$ of the quotient field of $R=R_{0}$. 
For $i \geq 0$, we will denote the set of proximate points of $R_{i}$ by

$$
\mathcal{P}\left(R_{i}\right)=\left\{R_{j} ; R_{j} \text { is proximate to } R_{i}\right\} .
$$

Note that $\mathcal{P}\left(R_{i}\right)$ is a finite set by statement 3) of Lemma 11. We will denote by

$$
n_{i}=\min \left\{v(z) ; z \in M\left(R_{i}\right)\right\}, \quad i \geq 0 .
$$

(Note that as $R_{i}$ is a noetherian ring then $n_{i}$ is well defined, $i \geq 0$ ).

Definition 14 With the above notations, the multiplicity sequence is given by

$$
\left\{\left(n_{0} / n_{i}, e_{i}\right)\right\}_{i \geq 1},
$$

where

$$
e_{i}=\left[R_{i} / M\left(R_{i}\right): R_{0} / M\left(R_{0}\right)\right],
$$

for $i \geq 1$. Note that $n_{0} / n_{i}$ is a real number with $1 \leq n_{0} / n_{i}, i \geq 1$.

Remark 15 If $z_{j} \in M\left(R_{j}\right)$ with $v\left(z_{j}\right)=n_{j}$, then

$$
R_{j+1}=\left(R_{j}\left[z_{j}^{-1} M\left(R_{j}\right)\right]\right)_{\mathfrak{q}_{j}},
$$

with $\mathfrak{q}_{j}$ a prime ideal of $R_{j}\left[z_{j}^{-1} M\left(R_{j}\right)\right]$ such that $M\left(R_{j}\right) R_{j}\left[z_{j}^{-1} M\left(R_{j}\right)\right] \subset \mathfrak{q}_{j}$.

In particular, $z_{j} \in M\left(R_{j+1}\right)-\{0\}$ and $n_{j+1} \leq n_{j}$, for all $j \geq 0$.

Lemma 16 With the above notations, we have $n_{i+1}=n_{i}$ if and only if $R_{i+2} \notin \mathcal{P}\left(R_{i}\right), i \geq 0$.

Proof: Let us write $M\left(R_{i}\right) R_{i+1}=z_{i} R_{i+1}$, with $v\left(z_{i}\right)=n_{i}>0$.

Let $E_{i}^{i+1}=\left(R_{i+1}, D_{i}^{i+1}\right)$ be the exceptional divisor attached to $R_{i}$ and $E_{i}^{j}=\left(R_{j}, D_{i}^{j}\right)$ its strict transform in $R_{j}, i<j$. We have $D_{i}^{i+1}=z_{i} R_{i+1}$.

Now, if $n_{i+1}=n_{i}$ then $D_{i}^{i+2}=R_{i+2}$ and $R_{i+2} \notin \mathcal{P}\left(R_{i}\right)$.

On the other hand, if $n_{i+1}<n_{i}$, then there exists $z_{i+1} \in M\left(R_{i+1}\right)$ with $v\left(z_{i+1}\right)=n_{i+1}$. Thus,

$$
D_{i}^{i+2}=\frac{z_{i}}{z_{i+1}} R_{i+2} \neq R_{i+2}
$$

and $R_{i+2} \in \mathcal{P}\left(R_{i}\right)$.

Lemma 17 With the above notations, we have

$$
n_{i}=\sum_{R_{j} \in \mathcal{P}\left(R_{i}\right)} n_{j}, \quad i \geq 0
$$


Proof: Let us consider

$$
h(i)=\max \left\{k ; R_{i+k} \in \mathcal{P}\left(R_{i}\right)\right\}, \quad i \geq 0 .
$$

We have $1 \leq h(i)$ and $R_{i+k} \in \mathcal{P}\left(R_{i}\right), 1 \leq k \leq h(i)$.

Let $z_{i+l} \in M\left(R_{i+l}\right)$ be such that $v\left(z_{i+l}\right)=n_{i+l}, 0 \leq l \leq h(i)$. Also let $E_{i}^{i+1}=\left(R_{i+1}, D_{i}^{i+1}\right)$ be the exceptional divisor attached to $R_{i}$ and $E_{i}^{j}=$ $\left(R_{j}, D_{i}^{j}\right)$ its strict transform in $R_{j}, i<j$.

We have $D_{i}^{i+1}=z_{i} R_{i+1}$ and

$$
D_{i}^{i+k}=\frac{z_{i}}{z_{i+1} \cdots z_{i+k-1}} R_{i+k}, \quad 1<k \leq h(i) .
$$

As $R_{i+h(i)+1} \notin \mathcal{P}\left(R_{i}\right)$, then

$$
n_{i+h(i)}=v\left(\frac{z_{i}}{z_{i+1} \cdots z_{i+h(i)-1}}\right) .
$$

Thus

$$
n_{i}=\sum_{R_{j} \in \mathcal{P}\left(R_{i}\right)} n_{j}=n_{i+1}+n_{i+2}+\ldots+n_{i+h(i)} .
$$

Let

$$
\alpha=q_{0}+\frac{1}{q_{1}+\frac{1}{q_{2}+\cdots}}
$$

be a finite or infinite continued fraction. Let $\left[q_{0}\right]:=q_{0}$ and define recursively $\left[q_{0}, \ldots, q_{j}\right]=\left[q_{0}, q_{1}, \ldots, q_{j-1}+1 / q_{j}\right]$ for $j \geq 1$ (as long as $q_{j} \neq 0$ ). Then we have

$$
\alpha=\lim _{n \rightarrow \infty}\left[q_{0}, q_{1}, \ldots, q_{n}\right] .
$$

As usually, we will write $\alpha=\left[q_{0}, q_{1}, q_{2} \ldots\right]$

Theorem 18 With the above notations, the refined proximity matrix determines the multiplicity sequence and vice-versa.

Proof: First we will see that the multiplicity sequence determines the refined proximity matrix $P\left(R_{i}\right)$.

Let us consider

$$
h(i)=\max \left\{k ; n_{i+1}+n_{i+2}+\cdots+n_{i+k} \leq n_{i}\right\}, \quad i \geq 0 .
$$

Note that

$$
h(i)=\max \left\{k ; R_{i+k} \in \mathcal{P}\left(R_{i}\right)\right\} .
$$


We compute the row $i$ of $P\left(R_{i}\right)$ as follows: $p_{i j}=0$ if $j<i$ or $i+h(i)<j$, $p_{i i}=1$, and

$$
p_{i j}=-e_{j} / e_{i}
$$

for $i+1 \leq j \leq i+h(i)$.

Conversely the refined proximity matrix determines the multiplicity sequence. Let us consider

$$
h(i)=\max \left\{k ; p_{i, i+k} \neq 0\right\}, \quad i \geq 0 .
$$

Note that

$$
h(i)=\max \left\{k ; R_{i+k} \in \mathcal{P}\left(R_{i}\right)\right\}=\max \left\{k ; n_{i+1}+n_{i+2}+\cdots+n_{i+k} \leq n_{i}\right\}
$$

and

$$
n_{i}=n_{i+1}+\cdots+n_{i+h(i)}, \quad i \geq 0 .
$$

It is easily checked that

$$
e_{i}=(-1)^{i} p_{01} p_{12} \ldots p_{i-1 i}, \quad i \geq 1 .
$$

On the other hand, let us write

$$
\frac{n_{0}}{n_{i}}=\left[q_{0}^{i}, q_{1}^{i}, q_{2}^{i}, \ldots\right]
$$

the continued fraction associated to $n_{0} / n_{i}$.

To finish the proof we point out that $q_{0}^{i}, q_{1}^{i}, q_{2}^{i}, \ldots$ are determined by $i$ and the sequence $(h(i))_{i \geq 0}$.

The above is a consequence of Corollary 23 given in the next section for a more general situation.

\section{Technical results}

In this section, we will study sequences of real numbers with properties as the multiplicity sequence.

Let $\left(n_{i}\right)_{i \geq 0}$ be a fixed sequence of positive real numbers and $(h(i))_{i \geq 0}$ be a sequence of positive integers such that $h(i)>0$ and

$$
n_{i}=n_{i+1}+\cdots+n_{i+h(i)}, \quad i \geq 0 .
$$

Lemma 19 With the above notations, let us consider $A=\sum_{j=0}^{k} a_{j} n_{j}$ and $B=\sum_{j=0}^{l} b_{j} n_{j}$, such that $a_{j} \in\{0,1\}$ for $0 \leq j \leq k, a_{k} \neq 0, b_{j} \in\{0,1\}$ for $0 \leq j \leq l, b_{0}=\cdots=b_{k}=0$ and $b_{l} \neq 0$. Then we can write $A=\sum_{j=0}^{s} \alpha_{j} n_{j}$ and $B=\sum_{j=0}^{s} \beta_{j} n_{j}$ such that $\alpha_{j}, \beta_{j}$ are non-negative integers and either $\alpha_{j} \geq \beta_{j}$ for $0 \leq j \leq s$ or $\beta_{j} \geq \alpha_{j}$ for $0 \leq j \leq s$. Moreover, $s, \alpha_{0}, \ldots, \alpha_{s}$, $\beta_{0}, \ldots, \beta_{s}$ can be constructed by using only $a_{0}, \ldots, a_{k}, b_{0}, \ldots, b_{l}$, and the sequence $(h(i))_{i \geq 0}$. 
Proof: Let $j_{0}$ be the non-negative integer given by

$$
j_{0}=\min \left\{j ; 0 \leq j \leq l \text { and } b_{j} \neq 0\right\} .
$$

Note that $k<j_{0} \leq l$.

Let us consider $\left(r_{i}\right)_{i \geq 0}$, the sequence of non-negative integers given by $r_{0}=k$, and $r_{i+1}=r_{i}+h\left(r_{i}\right)$, for $i \geq 0$. We have

$$
r_{0}<r_{1}<\cdots<r_{i}<\cdots
$$

Let $r_{m}$ be such that $r_{m-1}<j_{0} \leq r_{m}$.

Let us write

$$
A=\sum_{j=0}^{r_{m}} a_{j}^{\prime} n_{j},
$$

where $a_{j}^{\prime}=a_{j}$ for $0 \leq j<k=r_{0}, a_{j}^{\prime}=1$ if $j \neq r_{i}, 0 \leq i<m$, $r_{0}=k \leq j \leq r_{m}$ and $a_{r_{i}}^{\prime}=0$ for $0 \leq i<m$. (Note that $a_{j}^{\prime} \in\{0,1\}$ for $\left.0 \leq j \leq r_{m}\right)$.

Let $\delta \geq 1$ be the positive integer given by

$$
\delta=\sum_{j=j_{0}}^{l} b_{j} .
$$

If $\delta=1$, then $j_{0}=l$. Thus the result follows by taking $s=r_{m}, \alpha_{j}=a_{j}^{\prime}$, $0 \leq j \leq s=r_{m}, \beta_{j_{0}}=1$ and $\beta_{j}=0$ for $j \neq j_{0}, 0 \leq j \leq s=r_{m}$.

If $\delta>1$, we consider $C=\sum_{j=j_{0}}^{r_{m}} b_{j} n_{j}, A^{\prime \prime}=\sum_{j=0}^{r_{m}} a_{j}^{\prime \prime} n_{j}$ and $B^{\prime \prime}=$ $\sum_{j=r_{m}+1}^{l} b_{j} n_{j}$, where $a_{j}^{\prime \prime}=a_{j}^{\prime}$ for $0 \leq j<j_{0}, a_{j}^{\prime \prime}=0$ if $b_{j} \neq 0$ for $j_{0} \leq j \leq r_{m}$ and $a_{j}^{\prime \prime}=a_{j}^{\prime}$ if $b_{j}=0$ for $j_{0} \leq j \leq r_{m}$. (Note that if $l<r_{m}$, then $B^{\prime \prime}=0$, $B=C, A=A^{\prime \prime}+C$ and there is nothing to do).

In any case, we have $A=A^{\prime \prime}+C$ and $B=B^{\prime \prime}+C$.

At this point, applying induction on $\delta$ we get $A^{\prime \prime}=\sum_{j=0}^{s} \alpha_{j}^{\prime \prime} n_{j}$ and $B^{\prime \prime}=$ $\sum_{j=0}^{s} \beta_{j}^{\prime \prime} n_{j}$ such that $\alpha_{j}^{\prime \prime}, \beta_{j}^{\prime \prime}$ are non-negative integers and either $\alpha_{j}^{\prime \prime} \geq \beta_{j}^{\prime \prime}$ for $0 \leq j \leq s$ or $\beta_{j}^{\prime \prime} \geq \alpha_{j}^{\prime \prime}$ for $0 \leq j \leq s$. (Note that we can assume $r_{m} \leq s$ ).

Now, the result follows by taking $\alpha_{j}=\alpha_{j}^{\prime \prime}$ (resp. $\left.\beta_{j}=\beta_{j}^{\prime \prime}\right)$, for $0 \leq j<j_{0}$ or $r_{m}<j \leq s, \alpha_{j}=\alpha_{j}^{\prime \prime}+b_{j}$ (resp. $\left.\beta_{j}=\beta_{j}^{\prime \prime}+b_{j}\right)$, for $j_{0} \leq j \leq r_{m}$.

Lemma 20 With the above notations, let us consider $A=n_{i}$ and $B=$ $\sum_{j=0}^{l} b_{j} n_{j}$, with $b_{j} \in\{0,1\}$ for $0 \leq j \leq l$ and $b_{l} \neq 0$. Then we can write $A=$ $\sum_{j=0}^{s} \alpha_{j} n_{j}$ and $B=\sum_{j=0}^{s} \beta_{j} n_{j}$ such that $\alpha_{j}, \beta_{j}$ are non-negative integers and either $\alpha_{j} \geq \beta_{j}$ for $0 \leq j \leq s$ or $\beta_{j} \geq \alpha_{j}$ for $0 \leq j \leq s$. Moreover, $s, \alpha_{0}, \ldots$, $\alpha_{s}, \beta_{0}, \ldots, \beta_{s}$ are determined by $i, b_{0}, \ldots, b_{l}$, and the sequence $(h(i))_{i \geq 0}$. 
Proof: Let us consider

$$
j_{0}=\min \left\{j ; 0 \leq j \leq l \text { and } b_{j} \neq 0\right\} .
$$

We have two possibilities:

(1) $j_{0} \leq i$. In this case, let $l_{1}$ be the non-negative integer given by

$$
l_{1}=\max \left\{j ; 0 \leq j \leq i \text { and } b_{j} \neq 0\right\} .
$$

We have $j_{0} \leq l_{1} \leq i$. If $l_{1}=i$ then there is nothing to do $\left(b_{i}=1\right)$.

Now, let us assume $l_{1}<i$ and consider the sequence of non-negative integers $\left(r_{k}\right)_{k \geq 0}$, given by $r_{0}=l_{1}$, and $r_{k+1}=r_{k}+h\left(r_{k}\right)$, for $k \geq 0$. We have

$$
r_{0}<r_{1}<\cdots<r_{k}<\cdots \text {. }
$$

Let $r_{m}$ be such that $r_{m-1}<i \leq r_{m}$.

We can write $\beta_{j}=b_{j}, 0 \leq j<l_{1}=r_{0}, r_{m}<j \leq l, \beta_{r_{k}}=0,0 \leq k<m$, $\beta_{j}=b_{j}+1, i \leq j \leq r_{m}$ and $\beta_{j}=1, l_{1} \leq j<i$ and $j \notin\left\{r_{0}, r_{1}, \ldots, r_{m-1}\right\}$. Note that $\beta_{i}=1$ and the result follows by taking $\alpha_{j}=0$ if $j \neq i$ and $\alpha_{i}=1$. (2) $j_{0}>i$. In this case, we can apply Lemma 19 to obtain the result.

Lemma 21 With the above notations, let us consider $A=\sum_{j=0}^{k} a_{j} n_{j}$ and $B=\sum_{j=0}^{l} b_{j} n_{j}$, with $a_{j}$ a non-negative integer for $0 \leq j \leq k, a_{k} \neq 0$, $b_{j} \in\{0,1\}$ for $0 \leq j \leq l$ and $b_{l} \neq 0$. Then we can write $A=\sum_{j=0}^{s} \alpha_{j} n_{j}$ and $B=\sum_{j=0}^{s} \beta_{j} n_{j}$ such that $\alpha_{j}, \beta_{j}$ are non-negative integers and either $\alpha_{j} \geq \beta_{j}$ for $0 \leq j \leq s$ or $\beta_{j} \geq \alpha_{j}$ for $0 \leq j \leq s$. Moreover, $s, \alpha_{0}, \ldots, \alpha_{s}$, $\beta_{0}, \ldots, \beta_{s}$ can be constructed by using only $a_{0}, \ldots, a_{k}, b_{0}, \ldots, b_{l}$, and the sequence $(h(i))_{i \geq 0}$.

Proof: Let $\delta \geq 1$ be the positive integer given by

$$
\delta=\sum_{j=0}^{k} a_{j} .
$$

If $\delta=1$ the result follows from Lemma 20. Let us assume $\delta>1$. We have three possibilities:

(1) $l=k$.

Let us consider $A^{\prime}=\sum_{j=0}^{k} a_{j}^{\prime} n_{j}$ and $B^{\prime}=\sum_{j=0}^{k} b_{j}^{\prime} n_{j}$ where $a_{j}^{\prime}=a_{j}$, $b_{j}^{\prime}=b_{j}$ for $0 \leq j<k=l$, and $a_{k}^{\prime}=a_{k}-1, b_{k}^{\prime}=b_{k}-1=0$.

Now, applying induction on $\delta$ we get $A^{\prime}=\sum_{j=0}^{s} \alpha_{j}^{\prime} n_{j}$ and $B^{\prime}=\sum_{j=0}^{s} \beta_{j}^{\prime} n_{j}$ such that $\alpha_{j}^{\prime}, \beta_{j}^{\prime}$ are non-negative integers and either $\alpha_{j}^{\prime} \geq \beta_{j}^{\prime}$ for $0 \leq j \leq s$ or $\beta_{j}^{\prime} \geq \alpha_{j}^{\prime}$ for $0 \leq j \leq s$. Note that we can assume $l=k \leq s$.

To have the result, we must only write $\alpha_{j}=\alpha_{j}^{\prime}\left(\operatorname{resp} . \beta_{j}=\beta_{j}^{\prime}\right), j \neq k$, $0 \leq j \leq s$ and $\alpha_{k}=\alpha_{k}^{\prime}+1$ (resp. $\beta_{k}=\beta_{k}^{\prime}+1$ ). 
(2) $l<k$.

In this case, we consider the sequence of non-negative integers $\left(r_{i}\right)_{i \geq 0}$, given by $r_{0}=l$, and $r_{i+1}=r_{i}+h\left(r_{i}\right)$, for $i \geq 0$. We have

$$
r_{0}<r_{1}<\ldots<r_{i}<\ldots
$$

Let $r_{m}$ be such that $r_{m-1}<k \leq r_{m}$.

We write $B=\sum_{j=0}^{r_{m}} b_{j}^{\prime} n_{j}$, where $b_{j}^{\prime}=b_{j}$ for $0 \leq j<l=r_{0}, b_{j}^{\prime}=1$ if $j \neq r_{i}, 0 \leq i<m, r_{0}=l \leq j \leq r_{m}$ and $b_{r_{i}}^{\prime}=0$ for $0 \leq i<m$. Note that $b_{j}^{\prime} \in\{0,1\}, 0 \leq j \leq r_{m}$ and $b_{k}^{\prime}=1$.

Now, consider $C=n_{k}, A^{\prime \prime}=\sum_{j=0}^{k} a_{j}^{\prime \prime} n_{j}$ and $B^{\prime \prime}=\sum_{j=0}^{r_{m}} b_{j}^{\prime \prime} n_{j}$, where $a_{j}^{\prime \prime}=a_{j}, 0 \leq j<k, a_{k}^{\prime \prime}=a_{k}-1, b_{j}^{\prime \prime}=b_{j}^{\prime}, j \neq k, 0 \leq j \leq r_{m}$ and $b_{k}^{\prime \prime}=b_{k}^{\prime}-1=0$.

We have $A=A^{\prime \prime}+C$ and $B=B^{\prime \prime}+C$.

At this point, applying induction on $\delta$ we get $A^{\prime \prime}=\sum_{j=0}^{s} \alpha_{j}^{\prime \prime} n_{j}$ and $B^{\prime \prime}=$ $\sum_{j=0}^{s} \beta_{j}^{\prime \prime} n_{j}$ such that $\alpha_{j}^{\prime \prime}, \beta_{j}^{\prime \prime}$ are non-negative integers and either $\alpha_{j}^{\prime \prime} \geq \beta_{j}^{\prime \prime}$ for $0 \leq j \leq s$ or $\beta_{j}^{\prime \prime} \geq \alpha_{j}^{\prime \prime}$ for $0 \leq j \leq s$. Note that we can assume $r_{m} \leq s$.

To have the result, we must only write $\alpha_{j}=\alpha_{j}^{\prime \prime}\left(\right.$ resp. $\left.\beta_{j}=\beta_{j}^{\prime \prime}\right), j \neq k$, $0 \leq j \leq s$ and $\alpha_{k}=\alpha_{k}^{\prime \prime}+1$ (resp. $\left.\beta_{k}=\beta_{k}^{\prime \prime}+1\right)$.

(3) $l>k$.

In this case, let $j_{1}$ be the non-negative integer given by

$$
j_{1}=\min \left\{j ; \quad b_{j} \neq 0 \text { and } j \geq k\right\} .
$$

Note that $k \leq j_{1} \leq l$.

If $k=j_{1}$ let us consider $A^{\prime}=\sum_{j=0}^{k} a_{j}^{\prime} n_{j}$ and $B^{\prime}=\sum_{j=0}^{l} b_{j}^{\prime} n_{j}$ where $a_{j}^{\prime}=a_{j}, b_{j}^{\prime}=b_{j}$ if $j \neq j_{1}, a_{j_{1}}^{\prime}=a_{j_{1}}-1$ and $b_{j_{1}}^{\prime}=b_{j_{1}}-1=0$.

At this point, applying induction on $\delta$ we get $A^{\prime}=\sum_{j=0}^{s} \alpha_{j}^{\prime} n_{j}$ and $B^{\prime}=$ $\sum_{j=0}^{s} \beta_{j}^{\prime} n_{j}$ such that $\alpha_{j}^{\prime}, \beta_{j}^{\prime}$ are non-negative integers and either $\alpha_{j}^{\prime} \geq \beta_{j}^{\prime}$ for $0 \leq j \leq s$ or $\beta_{j}^{\prime} \geq \alpha_{j}^{\prime}$ for $0 \leq j \leq s$. Note that we can assume $l \leq s$.

To have the result, we must only write $\alpha_{j}=\alpha_{j}^{\prime}\left(\right.$ resp. $\left.\beta_{j}=\beta_{j}^{\prime}\right), j \neq j_{1}$, $0 \leq j \leq s$ and $\alpha_{j_{1}}=\alpha_{j_{1}}^{\prime}+1$ (resp. $\beta_{j_{1}}=\beta_{j_{1}}^{\prime}+1$ ).

Now, let us assume $k<j_{1} \leq l$ and consider the sequence of non-negative integers $\left(r_{i}\right)_{i \geq 0}$, given by $r_{0}=k$, and $r_{i+1}=r_{i}+h\left(r_{i}\right)$, for $i \geq 0$. We have

$$
r_{0}<r_{1}<\cdots<r_{i}<\cdots .
$$

Let $r_{m}$ be such that $r_{m-1}<j_{1} \leq r_{m}$.

We can write $A=\sum_{j=0}^{r_{m}} a_{j}^{\prime} n_{j}$, where $a_{j}^{\prime}=a_{j}, 0 \leq j<k=r_{0}, a_{k}^{\prime}=$ $a_{r_{0}}^{\prime}=a_{k}-1, a_{j}^{\prime}=1$ if $j \neq r_{i}, 0<i<m, r_{0}=k<j \leq r_{m}$ and $a_{r_{i}}^{\prime}=0$ for $0 \leq i \leq m-1$. (Note that $a_{j}^{\prime} \in\{0,1\}$ for $k<j \leq r_{m}$ ). 
Let us write $C^{1}=\sum_{j=j_{1}}^{r_{m}} b_{j} n_{j}, A^{1}=\sum_{j=0}^{r_{m}} a_{j}^{1} n_{j}$ and $B^{1}=\sum_{j=0}^{l} b_{j}^{1} n_{j}$, where $a_{j}^{1}=a_{j}^{\prime}, 0 \leq j<j_{1}, a_{j}^{1}=0$ if $b_{j} \neq 0, j_{1} \leq j \leq r_{m}, a_{j}^{1}=a_{j}^{\prime}$ if $b_{j}=0, j_{1} \leq j \leq r_{m}, b_{j}^{1}=b_{j}, 0 \leq j<j_{1}, b_{j}^{1}=0, j_{1} \leq j \leq r_{m}$ and $b_{j}^{1}=b_{j}$, $r_{m}<j \leq l$. (Here $b_{j}=0$ if $l<j \leq r_{m}$ ).

We have $A=A^{1}+C^{1}$ and $B=B^{1}+C^{1}$. If $B^{1}=0$, then there is nothing to do.

If $a_{j}^{1}=0$ for $j>k$, then $\sum_{j=0}^{r_{m}} a_{j}^{1}=\delta-1$ and applying induction on $\delta$ we get $A^{1}=\sum_{j=0}^{s} \alpha_{j}^{1} n_{j}$ and $B^{1}=\sum_{j=0}^{s} \beta_{j}^{1} n_{j}$ such that $\alpha_{j}^{1}, \beta_{j}^{1}$ are non-negative integers and either $\alpha_{j}^{1} \geq \beta_{j}^{1}$ for $0 \leq j \leq s$ or $\beta_{j}^{1} \geq \alpha_{j}^{1}$ for $0 \leq j \leq s$. We can assume $s \geq r_{m}$.

To have the result, we must only write $\alpha_{j}=\alpha_{j}^{1}\left(\operatorname{resp} . \beta_{j}=\beta_{j}^{1}\right)$ for $0 \leq j<j_{1}$ or $r_{m}<j \leq s$ and $\alpha_{j}=\alpha_{j}^{1}+b_{j}$ (resp. $\beta_{j}=\beta_{j}^{1}+b_{j}$ ) for $j_{1} \leq j \leq r_{m}$.

Let us assume $a_{j}^{1} \neq 0$ for some $j>k$ and $B^{1} \neq 0$. We have two possibilities:

a) $l \leq r_{m}$.

In this case, $b_{j}^{1}=0$ for $j \geq k$. Let us write

$$
j_{2}=\min \left\{j ; \quad a_{j}^{1} \neq 0 \text { and } j>k\right\}
$$

and

$$
l_{2}=\max \left\{j ; \quad b_{j}^{1} \neq 0\right\} .
$$

We have $l_{2}<k<j_{2}$.

Now, we consider the sequence of non-negative integers $\left(r_{i}^{1}\right)_{i \geq 0}$, given by $r_{0}^{1}=l_{2}$, and $r_{i+1}^{1}=r_{i}^{1}+h\left(r_{i}^{1}\right)$, for $i \geq 0$. We have

$$
r_{0}^{1}<r_{1}^{1}<\cdots<r_{i}^{1}<\cdots .
$$

Let $r_{m_{1}}^{1}$ be such that $r_{m_{1}-1}^{1}<j_{2} \leq r_{m_{1}}^{1}$.

We can write $B^{1}=\sum_{j=0}^{r_{m_{1}}^{1}} b_{j}^{\prime} n_{j}$, where $b_{j}^{\prime}=b_{j}^{1}, 0 \leq j<l_{2}=r_{0}^{1}, b_{r_{i}^{1}}^{\prime}=0$, $0 \leq i \leq m_{1}-1$ and $b_{j}^{\prime}=1, l_{2} \leq j \leq r_{m_{1}}^{1}, j \notin\left\{r_{0}^{1}, \ldots, r_{m_{1}-1}^{1}\right\}$. Note that $b_{j}^{\prime} \in\{0,1\}$ for $0 \leq j \leq r_{m_{1}}^{1}$.

Let us write $C^{2}=\sum_{j=j_{2}}^{r_{m_{1}}^{1}} a_{j}^{1} n_{j}, A^{2}=\sum_{j=0}^{r_{m}} a_{j}^{2} n_{j}$ and $B^{2}=\sum_{j=0}^{r_{m_{1}}^{1}} b_{j}^{2} n_{j}$, where $b_{j}^{2}=b_{j}^{\prime}, 0 \leq j<j_{2}, b_{j}^{2}=0$ if $a_{j}^{1} \neq 0, j_{2} \leq j \leq r_{m_{1}}^{1}, b_{j}^{2}=b_{j}^{\prime}$ if $a_{j}^{1}=0$, $j_{2} \leq j \leq r_{m_{1}}^{1}, a_{j}^{2}=a_{j}^{1}, 0 \leq j<j_{2}, a_{j}^{2}=0, j_{2} \leq j \leq r_{m_{1}}^{1}$ and $a_{j}^{2}=a_{j}^{1}$, $r_{m_{1}}^{1}<j \leq r_{m}$. (Here $a_{j}^{1}=0$ if $r_{m}<j \leq r_{m_{1}}^{1}$ ). 
We have $A^{1}=A^{2}+C^{2}$ and $B^{1}=B^{2}+C^{2}$. At this point, we have also two possibilities:

a.i) $r_{m} \leq r_{m_{1}}^{1}$. In this case, $a_{j}^{2}=0$ for $j>k$. Then $\sum_{j=0}^{r_{m}} a_{j}^{2}=\delta-1$ and applying induction on $\delta$ as above we get the Lemma.

a.ii) $r_{m}>r_{m_{1}}^{1}$. In this case, we can repeat in a similar way the reasoning of a). After a finite number, say $d$, of steps we get to one of the following statements:

a) $r_{m} \leq r_{m_{d}}^{d}$. In this situation, the Lemma follows by induction hypothesis on $\delta$ as in the case a.i).

B) $A=A^{d}+C^{1}+\cdots+C^{d}$ and $B=B^{d}+C^{1}+\cdots+C^{d}$, with $B^{d}=0$. In this case, we also get the Lemma by taking expressions of $A^{d}$, $C^{1}, \ldots, C^{d}$.

b) $l>r_{m}$

In this case, let us write

$$
j_{2}=\min \left\{j ; b_{j}^{1} \neq 0 \text { and } j>r_{m}\right\} \quad \text { and } \quad k_{2}=\max \left\{j ; a_{j}^{1} \neq 0\right\} .
$$

We have $k<k_{2} \leq r_{m}<j_{2}$.

Now, we consider the sequence of non-negative integers $\left(r_{i}^{1}\right)_{i>0}$, given by $r_{0}^{1}=k_{2}$, and $r_{i+1}^{1}=r_{i}^{1}+h\left(r_{i}^{1}\right)$, for $i \geq 0$. We have

$$
r_{0}^{1}<r_{1}^{1}<\cdots<r_{i}^{1}<\cdots .
$$

Let $r_{m_{1}}^{1}$ be such that $r_{m_{1}-1}^{1}<j_{2} \leq r_{m_{1}}^{1}$.

We can write $A=\sum_{j=0}^{r_{m_{1}}^{1}} a_{j}^{\prime \prime} n_{j}$, where $a_{j}^{\prime \prime}=a_{j}^{1}, 0 \leq j<k_{2}=r_{0}^{1}, a_{r_{i}^{1}}^{\prime \prime}=0$, $0 \leq i \leq m_{1}-1$ and $a_{j}^{\prime \prime}=1, k_{2} \leq j \leq r_{m_{1}}^{1}, j \notin\left\{r_{0}^{1}, \ldots, r_{m_{1}-1}^{1}\right\}$. Note that $a_{j}^{\prime \prime} \in\{0,1\}$ for $k<j \leq r_{m_{1}}^{1}$.

Let us write $C^{2}=\sum_{j=j_{2}}^{r_{m_{1}}^{1}} b_{j}^{1} n_{j}, A^{2}=\sum_{j=0}^{r_{m_{1}}^{1}} a_{j}^{2} n_{j}$ and $B^{2}=\sum_{j=0}^{l} b_{j}^{2} n_{j}$, where $a_{j}^{2}=a_{j}^{\prime \prime}, 0 \leq j<j_{2}, a_{j}^{2}=0$ if $b_{j}^{1} \neq 0, j_{2} \leq j \leq r_{m_{1}}^{1}, a_{j}^{2}=a_{j}^{\prime \prime}$ if $b_{j}^{1}=0, j_{2} \leq j \leq r_{m_{1}}^{1}, b_{j}^{2}=b_{j}^{1}, 0 \leq j<j_{2}, b_{j}^{2}=0, j_{2} \leq j \leq r_{m_{1}}^{1}$ and $b_{j}^{2}=b_{j}^{1}$, $r_{m_{1}}^{1}<j \leq l$. (Here $b_{j}^{1}=0$ if $l<j \leq r_{m_{1}}^{1}$ ).

We have $A^{1}=A^{2}+C^{2}$ and $B^{1}=B^{2}+C^{2}$. At this point, we have also two possibilities:

b.i) $l \leq r_{m_{1}}^{1}$. In this case, $b_{j}^{2}=0$ for $j>k$. If $a_{j}^{2}=0$ for $j>k$, the result follows by induction on $\delta$ as above.

If there exists $a_{j}^{2} \neq 0$ for some $j>k$, we are in a similar situation as in the case a). So we also get the Lemma with a similar reasoning as case a). 
b.ii) $l>r_{m_{1}}^{1}$. In this case, we can repeat in a similar way the reasoning in b). After a finite number, say $d$, of steps we get $l \leq r_{m_{d}}^{d}$. Therefore, we get the Lemma in a similar way as case b.i).

Theorem 22 With the above notations, let us consider $A=\sum_{j=0}^{k} a_{j} n_{j}$ and $B=\sum_{j=0}^{k} b_{j} n_{j}$, with $a_{j}$ and $b_{j}$ non-negative integers for $0 \leq j \leq k$. If $A \geq B>0$ then we can write $A=\sum_{j=0}^{s} \alpha_{j} n_{j}$ and $B=\sum_{j=0}^{s} \beta_{j} n_{j}$ such that $\alpha_{j}, \beta_{j}$ are non-negative integers and $\alpha_{j} \geq \beta_{j} \geq 0$ for $0 \leq j \leq s$. Moreover, $s$, $\alpha_{0}, \ldots, \alpha_{s}, \beta_{0}, \ldots, \beta_{s}$ can be constructed by using only $a_{0}, \ldots, a_{k}, b_{0}, \ldots, b_{k}$, and the sequence $(h(i))_{i \geq 0}$.

Proof: Let $\delta \geq 1$ be the positive integer given by

$$
\delta=\sum_{j=0}^{k} b_{j} .
$$

If $\delta=1$, the result follows from Lemma 21. If $\delta>1$, let us assume $b_{i} \neq 0$.

By Lemma 21 applied to $A$ and $n_{i}$, we can write $n_{i}=\sum_{j=0}^{k} b_{j}^{*} n_{j}$, such that $a_{j}, b_{j}^{*}$ are non-negative integers and $a_{j} \geq b_{j}^{*}$ for $0 \leq j \leq k$. Note that $A \geq B \geq n_{i}>0$.

We write $A^{\prime}=\sum_{j=0}^{k} a_{j}^{\prime} n_{j}$ and $B^{\prime}=\sum_{j=0}^{k} b_{j}^{\prime} n_{j}$, with $a_{j}^{\prime}=a_{j}-b_{j}^{*}, 0 \leq$ $j \leq k, b_{j}^{\prime}=b_{j}$ if $j \neq i$ and $b_{i}^{\prime}=b_{i}-1$.

As $\sum_{j=0}^{k} b_{j}^{\prime}=\delta-1$, applying induction on $\delta$ we get $A^{\prime}=\sum_{j=0}^{s} \alpha_{j}^{\prime} n_{j}$ and $B^{\prime}=\sum_{j=0}^{s} \beta_{j}^{\prime} n_{j}$ such that $\alpha_{j}^{\prime}, \beta_{j}^{\prime}$ are non-negative integers and $\alpha_{j}^{\prime} \geq \beta_{j}^{\prime}$ for $0 \leq j \leq s$. Note that $A^{\prime} \geq B^{\prime}>0$ and we can assume $s \geq k$.

To obtain the result we must only write $\alpha_{j}=\alpha_{j}^{\prime}+b_{j}^{*}\left(\operatorname{resp} . \beta_{j}=\beta_{j}^{\prime}+b_{j}^{*}\right)$ for $0 \leq j \leq s$. (Here $b_{j}^{*}=0$ for $k<j \leq s$ ).

Corollary 23 With the above notations, let us consider $A=\sum_{j=0}^{k} a_{j} n_{j}$ and $B=\sum_{j=0}^{k} b_{j} n_{j}$, with $a_{j}, b_{j}$ non-negative integers for $0 \leq j \leq k$ and $B \neq 0$.

Let $A / B=\left[q_{0}, q_{1}, q_{2} \ldots\right]$ be the continued fraction associated to $A / B$. Then $q_{0}, q_{1}, \ldots$ can be constructed by using only $a_{0}, \ldots, a_{k} b_{0}, \ldots, b_{k}$, and the sequence $(h(i))_{i \geq 0}$.

Proof: If $A \geq B$ then by Theorem 22 we can assume that $a_{j}, b_{j}$ are nonnegative integers and $a_{j} \geq b_{j}$ for $0 \leq j \leq k$, and $b_{j}>0$ for some $j$. In this case

$$
\frac{A}{B}=1+\frac{A^{1}}{B},
$$

with $A^{1}=\sum_{j=0}^{k} a_{j}^{1} n_{j}$ and $a_{j}^{1}=a_{j}-b_{j}, 0 \leq j \leq k$.

If $A^{1} \geq B$, by Theorem 22 , we can assume that $a_{j}^{1} \geq b_{j}$ for $0 \leq j \leq k$. 
Now, we can repeat the process with $A^{1} / B$ and after $q_{0}$ steps we get

$$
\frac{A}{B}=q_{0}+\frac{A^{q_{0}}}{B}=q_{0}+\frac{1}{\frac{B}{A^{q_{0}}}},
$$

with $A^{q_{0}}=\sum_{j=0}^{k} a_{j}^{q_{0}} n_{j}, a_{j}^{q_{0}}$ a non-negative integer for $0 \leq j \leq k$ and $B>A^{q_{0}}$.

If $A<B$ then $q_{0}=0$ and we shall take $A^{q_{0}}=A$ to have

$$
\frac{A}{B}=0+\frac{1}{\frac{B}{A^{q_{0}}}},
$$

and $B>A^{q_{0}}$.

At this point, we can use induction to obtain the result in any case.

\section{References}

[1] Abhyankar, S. S.: On the valuations centered in a local domain. Amer. J. Math. 78 (1956), 321-348.

[2] Abhyankar, S.S.: Desingularization of plane curves. In Singularities, Part 1 (Arcata, Calif., 1981), 1-45. Proc. Sympos. Pure Math. 40. Amer. Math. Soc., Providence, RI, 1983.

[3] Abhyankar, S. S.: Good points of a hypersurface. Adv. in Math. 68 (1988), 87-256.

[4] Abhyankar, S.S.: Algebraic Geometry for Scientists and Engineers. Mathematical Surveys and Monographs 35. American Mathematical Society, Providence, RI, 1990.

[5] Abhyankar, S. S.: Ramification Theoretic Methods in Algebraic Geometry. Annals of Mathematics Studies 43. Princeton University Press, Princeton, N.J. 1959.

[6] Abhyankar, S. S.: Resolution of Singularities of Embedded Algebraic Surfaces. Springer-Verlag. New York Inc., 1998.

[7] Abhyankar, S. S.: Resolution of singularities and modular Galois theory. Bull. Amer. Math. Soc. (N.S.) 38 (2001), no. 2, 131-169.

[8] Aparicio, J., Granja, A. and Sánchez-Giralda, T.: On proximity relations for valuations dominating a two-dimensional regular local ring. Rev. Mat. Iberoamericana 15 (1999), no. 3, 621-634.

[9] Delgado, D., Galindo, C. And Núñez, A.: Saturation for valuations on two-dimensional regular rings. Math. Z. 234 (2000), 519-550.

[10] Granja, A. and SÁnchez-Giralda, T.: Enriques graphs of plane curves. Comm. Algebra 20 (1992), no. 2, 527-562. 
[11] Lipman, J.: Proximity inequalities for complete ideals in two-dimensional regular local rings. In Commutative algebra: syzygies, multiplicities, and birational algebra (South Hadley, MA, 1992), 293-306. Contemp. Math. 159. Amer. Math. Soc., Providence, RI, 1994.

[12] Nagata, M.: Local Rings. Interscience Tracts in Pure and Applied Mathematics 13, Interscience Publishers, a division of John Wiley \& Sons, New York-London, 1962.

[13] Shannon, D. L.: Monoidal transforms of regular rings. Amer. J. Math. 95 (1973), 294-320.

[14] Vaquié, M.: Valuations. In Resolution of singularities (Obergurgl, 1997), 539-590. Progr. Math. 181, Birkhäuser, Basel, 2000.

[15] Zariski, O.: Local uniformization on algebraic varieties. Ann. of Math. 41 (1940), 852-896.

[16] Zariski, O. and Samuel, P.: Commutative Algebra, Vols. I and II. D. Van Nostrand Company, Inc., Princeton, New Jersey, 1958, 1960.

Recibido: 3 de abril de 2002

Revisado: 8 de agosto de 2002

Ángel Granja

Department of Mathematics

University of León

Campus de Vegazana, s/n. 24071-León, Spain

demagb@unileon.es

Cristina Rodríguez

Department of Mathematics

University of León

Campus de Vegazana, s/n. 24071-León, Spain

demmrs@unileon.es

Both authors are partially supported by DGICYT, PB98-0753-C02-01. 